\title{
Would Hydroxychloroquine Be A New Promising Drug in Managing Antiphospholipid Syndrome?
}

\author{
Amani Mohsen ${ }^{1 *}$ and Rabih Chahine ${ }^{2}$ \\ ${ }^{1}$ Obstetrics \& gynecology consultant, Palestinian Red Crescent Society, Shatila Camp, Beirut, Lebanon \\ ${ }^{2}$ chairman of obstetrics \& gynecology department, Rafik Hariri university Hospital, Beirut, Lebanon
}

Received: 眥 October 10, 2018; Published: 監 October 15, 2018

*Corresponding author: Amani Mohsen, Obstetrics \& gynecology consultant, Palestinian Red Crescent Society, Shatila Camp, Beirut, Lebanon

\begin{abstract}
Once Antiphospholipid syndrome (APS) is diagnosed, risk profile of the patient will impact its management whether there was a previous thrombotic event or not, association with other autoimmune disorders, presence of other obstetrical complications as well as being currently pregnant. The current recommended regimen for preventing obstetrical complications. The current recommended regimen for preventing obstetrical complications includes low molecular weight Heparin and low dose aspirin. This regimen decreases the risk of miscarriage by only 54\%. Here, we will review the studies that evaluated adding Hydroxychloroquine (HCQ) to the treatment of APS and how effective it would be.
\end{abstract}

Keywords: Antiphospholipid syndrome; Hydroxychloroquine; Miscarriage

\section{Introduction}

Antiphospholipid syndrome is an autoimmune disorder defined by the presence of either vascular thrombosis or characteristic obstetrical morbidities along with positive laboratory tests of circulating antibodies. Women with APS would present with pregnancy morbidities including three recurrent miscarriages before 10th week or unexplained stillbirth [1]. Once diagnosis is confirmed, management of APS is warranted to prevent obstetrical morbidities and risk of thrombosis which would reach $25 \%$ during pregnancy and the postpartum period. Long term follow- up revealed that $50 \%$ of women with APS but without thrombotic events would develop vascular thrombosis during 3-10 years and $10 \%$ would develop systematic lupus erythematosus (SLE) [2]. Different guidelines recommend prophylactic heparin during pregnancy and the postpartum period to prevent risk of thrombosis and increase the rate of live births $[1,3]$. In a Cochrane review of randomized trials, combination of low dose aspirin and heparin decreases the risk of miscarriage by 54\% [4]. Without any pharmacologic treatment, the live birth rate would reach only $10 \%$ in women suffering from APS with recurrent miscarriage [5].

Looking for other medications that would decrease risk of thrombosis, Hydroxychloroquine (HCQ) was historically evaluated to play this role. HCQ is an anti-malarial medication which is used to treat autoimmune rheumatic conditions gaining benefit from its ability to boost immune system against self-antigens [6]. In 1970s, placebo controlled randomized trials examined HCQ as a promising agent to prevent postoperative venous thrombosis in fields of general and orthopedic surgeries, but it failed [7,8]. Later, in a nested case control study of SLE patients with thrombosis matched with SLE controls without thrombosis, HCQ was associated with $68 \%$ decrease in risk of thrombosis [9]. This reduction was only borderline $(\mathrm{P}=0.05)$ in a case control study of SLE patients with positive APS but without previous thrombosis matched with SLE controls without APS [10]. In a small prospective cohort study of primary APS with previous venous thrombosis, HCQ was added to the oral anticoagulant $(\mathrm{OA})$ in 20 patients while the other 20 patients were treated with $\mathrm{OA}$ alone. Six cases of recurrences were documented in the group treated with $\mathrm{OA}$ alone versus no recurrence in the group treated with HCQ with OA. However, evidence from this study is limited due to small size of the cohort and selection bias where risk of relapses (30\%) is also high in the group receiving $\mathrm{OA}[11]$.

Mekinian et al. [12] addressed the role of HCQ in pregnant women with APS without baseline SLE in a retrospective cohort study, where both cases and controls received combination treatment of low molecular weight heparin (LMWH) and aspirin with the addition of HCQ to the cases. Pregnancy loss was 
significantly decreased from $81 \%$ to $19 \%$ [12]. This study was a multicenter study with a small sample size and more autoimmune diseases reported in the cases where prednisone was added also to their treatment regimen contributing to selection bias. The used LMWH was not clearly identified if it was the same among centers or different LMWH was used where pregnancy loss was very high in the control group although being treated with the recommended conventional regimen. Another retrospective study examined the role of adding HCQ to patients with APS during their pregnancy. In this study, HCQ was given to the group of patients with SLE while in the control group only 5 patients diagnosed with SLE and APS who refused HCQ during pregnancy. Results confirmed the safety profile of HCQ with higher rate of live birth (66.7\% versus $57.1 \%$, $\mathrm{P}=0.05$ ) and lower rate of pregnancy complications (47\% versus $63 \%, \mathrm{P}=0.004$ ) in the HCQ group. However, the group who received conventional treatment without HCQ had significantly higher previous pregnancy morbidities $(\mathrm{P}=0.004)$. Also, different baseline regimens of aspirin +/- prophylactic or therapeutic LMWH were given with or without HCQ [13]. Adding to the fact that HCQ was given to the group diagnosed with both SLE plus APS, selection bias is profound. So, results should be taken cautiously.

A recent small retrospective cohort study of patients diagnosed with APS without any related connective tissue disease evaluated the role of adding HCQ to the conventional treatment of LMWH. HCQ was started six months prior to gestation and continued all over the pregnancy. Patients received HCQ have higher birth rate than the control group ( $\mathrm{P}=0.05)$ [14]. The PREGNANTS study determined the severity of APS depending on the underlying positive antiphospholipid antibody. Being treated with combination of prophylactic LMWH and low dose aspirin, the rate of live birth was $79.6 \%$ if only lupus anticoagulant is positive, $56.3 \%$ if only anticardiolipin is positive, $47.7 \%$ if anti- $\beta 2$ glycoprotein-I only is positive, $43.3 \%$ if double positive and lupus anticoagulant is negative and $30 \%$ if triple positive [15]. In case of severe APS with previous pregnancy morbidities, adding HCQ to the combination treatment would be a credit waiting the evidence expected from "HYPATIA" randomized controlled trial [16] which is planned to evaluate the pregnancy outcomes after adding HCQ to the standard treatment. In conclusion, management of APS should be considered in light of previous thrombotic event, the risk associated with the underlying positive antiphospholipid antibodies and adverse obstetrical events. Adding Hydroxychloroquine to the standard regimen of LMWH and low dose aspirin would be highly promising in severe APS cases waiting further evidence from HYPATIA study.

\section{References}

1. Committee on Practice Bulletins-Obstetrics A (2012) Practice Bulletin No. 132: Antiphospholipid syndrome. Obstetrics and gynecology 120(6): 1514-1521.
2. Silver RM, Draper ML, Scott JR, Lyon JL, Reading J, et al. (1994) Clinical consequences of antiphospholipid antibodies: an historic cohort study. Obstetrics and gynecology 83(3): 372-377.

3. (2011) The investigation and treatment of couples with recurrent first-trimester and second-trimester miscarriage. Royal College of Obstetricians and Gynaecologists.

4. Empson M, Lassere M, Craig J, Scott J (2005) Prevention of recurrent miscarriage for women with antiphospholipid antibody or lupus anticoagulant. (2): CD002859.

5. Rai RS, Clifford K, Cohen H, Regan L (1995) High prospective fetal loss rate in untreated pregnancies of women with recurrent miscarriage and antiphospholipid antibodies. Human reproduction 10(12): 3301-3304.

6. Rainsford KD, Parke AL, Clifford-Rashotte M, Kean WF (2015) Therapy and pharmacological properties of hydroxychloroquine and chloroquine in treatment of systemic lupus erythematosus, rheumatoid arthritis and related diseases. Inflammopharmacology 23(5): 231-269.

7. Cooke ED, Dawson MH, Ibbotson RM, Bowcock SA, Ainsworth ME, et al. (1977) Failure of orally administered hydroxychloroquine sulphate to prevent venous thromboembolism following elective hip operations. The Journal of bone and joint surgery American volume 59(4): 496-500.

8. Petri M (2011) Use of Hydroxychloroquine to prevent thrombosis in systemic lupus erythematosus and in antiphospholipid antibodypositive patients. Current rheumatology reports 13(1): 77-80.

9. Jung H, Bobba R, Su J, Shariati-Sarabi Z, Gladman DD, et al. (2010) The protective effect of antimalarial drugs on thrombovascular events in systemic lupus erythematosus. Arthritis \& Rheumatism 62(3): 863-868.

10. Tektonidou MG, Laskari K, Panagiotakos DB, Moutsopoulos HM (2009) Risk factors for thrombosis and primary thrombosis prevention in patients with systemic lupus erythematosus with or without antiphospholipid antibodies. Arthritis Care \& Research 61(1): 29-36.

11. Schmidt-Tanguy A, Voswinkel J, Henrion D, Subra JF, Loufrani L, et al. (2013) Antithrombotic effects of hydroxychloroquine in primary antiphospholipid syndrome patients. Journal of Thrombosis and Haemostasis 11(10): 1927-1929.

12. Mekinian A, Lazzaroni MG, Kuzenko A, Alijotas-Reig J, Ruffatti A, et al. (2015) The efficacy of hydroxychloroquine for obstetrical outcome in anti-phospholipid syndrome: data from a European multicenter retrospective study. Autoimmunity reviews 14(6): 498-502.

13. Sciascia S, Hunt BJ, Talavera-Garcia E, Lliso G, Khamashta MA, et al. (2016) The impact of hydroxychloroquine treatment on pregnancy outcome in women with antiphospholipid antibodies. American journal of obstetrics and gynecology 214(2): e1-273-e8.

14. Sciascia S, Baldovino S, Roccatello D, Cuadrado MJ, (2016) Role of Hydroxychloroquine in Improving Pregnancy Outcomes in Women with Antiphospholipid Antibodies without Other Underlying Connective Tissue Disease, New Jersey, USA.

15. Saccone G, Maruotti GM, Berghella V, Sarno L, Capone A, et al. (2017) 62: Obstetric outcomes in pregnant women with primary antiphospholipid syndrome according to the antibody profile: The PREGNANTS study. American Journal of Obstetrics \& Gynecology 216(1): S45-S46.

16. Schreiber K, Breen K, Cohen H, Jacobsen S, Middeldorp S, et al. (2017) HYdroxychloroquine to Improve Pregnancy Outcome in Women with AnTIphospholipid Antibodies (HYPATIA) protocol: a multinational randomized controlled trial of hydroxychloroquine versus placebo in addition to standard treatment in pregnant women with antiphospholipid syndrome or antibodies, Thieme Medical Publishers, Germany, 43(6): 562-571. 


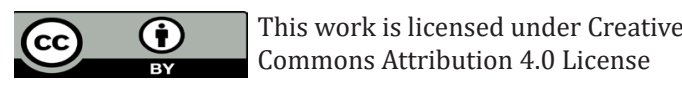

Submission Link:

Submit Article

DOI: 10.32474/DDIPIJ.2018.02.000141

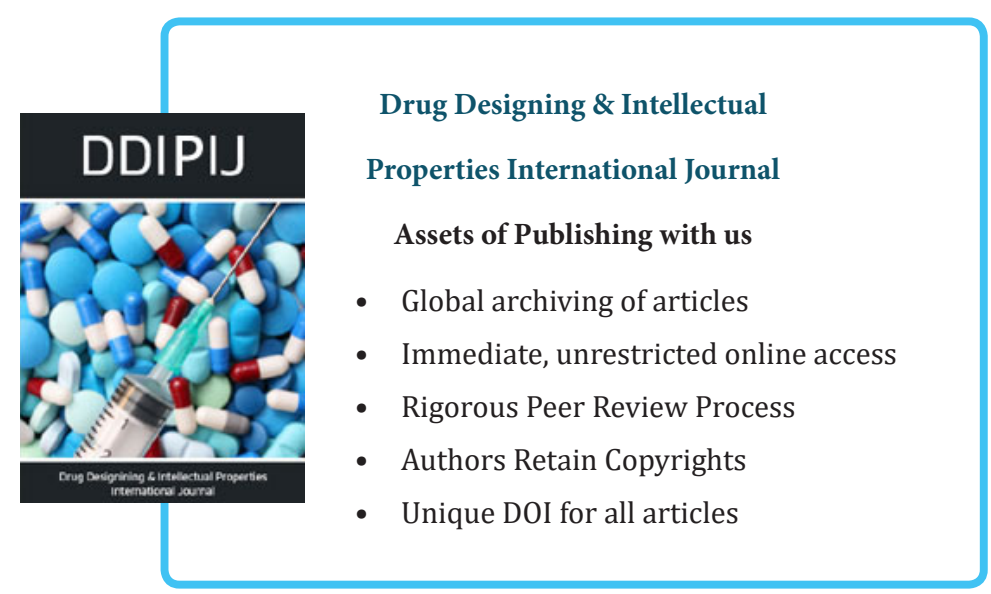

